Le point sur...

\title{
Angleterre : le A-Level en question
}

\section{Cécile de Bouttemont}

\section{CpenEdition}

\section{Journals}

Édition électronique

URL : https://journals.openedition.org/ries/1689

DOI : 10.4000/ries. 1689

ISSN : 2261-4265

Éditeur

France Education international

Édition imprimée

Date de publication : 1 avril 2003

Pagination : 14-16

ISSN : $1254-4590$

Référence électronique

Cécile de Bouttemont, «Angleterre : le A-Level en question », Revue internationale d'éducation de Sèvres [En ligne], 32 | avril 2003, mis en ligne le 01 mars 2006, consulté le 06 juillet 2021. URL : http:// journals.openedition.org/ries/1689; DOI : https://doi.org/10.4000/ries.1689

Ce document a été généré automatiquement le 6 juillet 2021.

(C) Tous droits réservés 


\title{
Angleterre : le A-Level en question
}

\author{
Cécile de Bouttemont
}

1 La publication en août dernier des résultats du A-level, examen sanctionnant la fin des études secondaires et ouvrant accès à l'université, a débouché sur une crise sans précédent. À la suite de nombreuses plaintes d'élèves, les responsables des principaux centres d'examens du royaume ont reconnu avoir, à cette occasion, délibérément sousnoté certains candidats afin de maintenir le prestige de l'examen face à des résultats jugés trop favorables. L'enjeu est de taille puisque les premières estimations faisaient état de 90000 candidats $^{1}$ potentiellement affectés, ce qui pouvait empêcher certains d'entre eux d'entrer à l'université de leur choix.

2 Créé en 1944 par la loi Butler et réactivé par la réforme de 1988, le A-level se prépare en deux ans. Les élèves choisissent généralement quatre matières en première année et obtiennent pour chaque matière une note globale intégrant les examens de première et deuxième années ainsi que le contrôle continu. Réussir son A-level consiste à obtenir une bonne note en deuxième année dans au moins trois matières sur les quatre sélectionnées. C'est sur cette base que les universités effectuent leur recrutement.

3 Pour la passation des épreuves, chaque établissement du secondaire choisit l'un des trois centres d'examens que compte l'Angleterre. Il s'agit d'institutions privées placées sous le contrôle d'un organisme d'État, le Qualifications and Curriculum Authority (QCA) qui veille au bon fonctionnement du système. Elles sont chargés de la préparation des sujets d'examens et de leur correction. Les notes obtenues à chaque épreuve sont ensuite harmonisées et agrégées en une seule selon un processus complexe placé sous la responsabilité du centre d'examen, de façon à ce que les élèves soient toujours évalués suivant un standard commun d'année en année.

4 C'est ce processus qui a été vivement critiqué après la publication des résultats du Alevel 2002. Le centre d'examen $\mathrm{OCR}^{2}$ a par la suite reconnu avoir volontairement remonté les barèmes à la fin du processus de notation pour réduire le taux de réussite jusqu'à un niveau comparable à celui de 2001 afin de ne pas dévaloriser l'examen. 
5 Après la démission de la secrétaire d'État à l'Éducation et le remplacement du responsable du QCA, le gouvernement a décidé de lancer un processus de recorrection et de conduire une enquête approfondie pour améliorer le système.

Le rapport Tomlinson ${ }^{3}$ explique les différents dysfonctionnements observés par plusieurs facteurs, dont les plus importants sont :

- l'absence de directives claires de la part du QCA sur les standards à respecter et les difficultés à effectuer des comparaisons en l'absence de statistiques tenant compte des nouvelles modalités introduites par les réformes de $2000^{4}$;

- l'engorgement du système (manque de moyens face à l'inflation du nombre des candidats et des examens, délais de correction trop courts...), ce qui a conduit à prendre des décisions dans l'urgence concernant les barèmes au niveau des centres d'examens.

7 Par ailleurs, estimant que le système actuel souffre globalement d'une grande complexité et d'une certaine opacité, le rapporteur suggère différentes pistes d'amélioration du système actuel, qui doivent faire l'objet d'une étude plus approfondie.

Concernant les structures et les contenus, il recommande en particulier une définition plus précise des standards attendus par le QCA, ainsi qu'une formalisation des procédures de contrôle du processus de notation et du rôle des différents intervenants. L'évaluation du cursus pourrait en outre être facilitée par la séparation des deux années en deux examens distincts et non combinés au sein d'une même note, ainsi que par une simplification et une clarification des règles d'harmonisation des notes obtenues à chaque épreuve. Enfin, il suggère la professionnalisation de la fonction de correcteur par la mise de en place de formations spécifiques.

9 Le rapport propose également d'améliorer l'organisation et la gestion matérielle des examens en renforçant les moyens mis à la disposition des services chargés de les gérer, et en particulier les moyens informatiques, de réduire leur charge de travail en limitant le nombre des examens ${ }^{5}$, en augmentant la part du contrôle continu et en reportant la date des inscriptions à l'université afin de laisser plus de temps aux correcteurs.

10 Face au risque de voir le A-level complètement discrédité, le gouvernement a immédiatement décidé d'adopter les principales recommandations du rapport Tomlinson pour assurer le bon déroulement de la prochaine session (nomination d'un observateur externe chargé de contrôler le processus de notation, déblocage de 6 millions de livres ${ }^{6}$ afin de recruter 50000 correcteurs et déplacement de la date de certains examens).

11 En réalité, ce scandale a été l'occasion pour l'opposition de remettre en cause la réforme mise en place en 2000 et dans laquelle l'introduction du contrôle continu a fait l'objet d'un vif débat au sein de l'opinion publique. En effet les conservateurs reprochent globalement au gouvernement d'avoir ainsi contribué à abaisser le niveau global de cet examen et entraîné une surcharge de cette filière.

12 La résurgence des clivages politiques à propos de ce scandale est liée au véritable enjeu que constitue la pertinence et la validité de la certification de fin d'études secondaires. Ainsi, on reproche au A-level une spécialisation trop précoce et certains observateurs demandent la création d'un examen de type baccalauréat, intégrant un plus grand nombre de matières. De son côté, par l'intermédiaire du rapport Tomlinson, le 
gouvernement souhaite le maintien du système existant moyennant quelques adaptations ainsi que le renforcement du contrôle continu.

Enfin, des interrogations subsistent sur la finalité du A-level qui est utilisé, d'une part, comme un moyen de comparer le niveau des candidats par rapport à un standard minimal fixe et, d'autre part, comme un outil permettant aux universités de sélectionner les meilleurs candidats. Chacune des deux fonctions impose des caractéristiques spécifiques qui peuvent se révéler parfois contradictoires.

\section{NOTES}

1. Mike Tomlinson, Report on outcomes of A-level grading, 2 décembre 2002.

2. Oxford, Cambridge et RSA.3. Ancien inspecteur principal de l'enseignement.

3. Ancien inspecteur principal de l'enseignement.

4. Renforcement du système modulaire dans le A-level: l'AS-level correspondant à la première année et autrefois distinct du A-level devient le premier module nécessaire pour continuer en $2^{\mathrm{e}}$ année désormais appelée A2-level. Un AS plus un $\mathrm{A} 2$ réussis équivalent à un A-level.

5. Standard Assessment Tests à 14 ans, General Certificate of standard evaluation (GCSE) à 15 et 16 ans, AS-level à 17 ans et A-level à 18 ans.

6. Environ 9 millions d'euros.

\section{INDEX}

Index géographique : Angleterre, Royaume-Uni

Mots-clés : diplôme, enseignement secondaire

\section{AUTEUR}

\section{CÉCILE DE BOUTTEMONT}

Documentaliste, centre de ressources documentaires du CIEP 